\title{
Radiofrequency as a lesioning model in experimental spinal cord injury
}

\author{
Siavash S Haghighi ${ }^{1}$, Miguel-A Perez-Espejo ${ }^{1}$, Fabio Rodriguez ${ }^{2}$ and Adam Clapper ${ }^{1}$ \\ ${ }^{1}$ Division of Neurosurgery, and ${ }^{2}$ Department of Radiology, University of Missouri-Columbia, Columbia, Missouri \\ 65212, USA
}

\begin{abstract}
Many models have been developed to study spinal cord injury (SCI), such as cryogenic lesioning, hot water injury, scalpel lesioning, compressive trauma using clips, electromechanical devices, extradural cuffs, and weight-drop techniques. In this study, the radiofrequency (RF) lesion was used for inducing an experimental SCI in cats. The neuropathology was correlated with the MRI. In this model, 4 cats were injured at the thoracic spinal cord (T11$\mathrm{T} 12)$ with a lesion of $65^{\circ} \mathrm{C}$ for 1 min using a micromanipulated penetrating $\mathrm{RF}$ electrode. The MRI of the lesions after 2, 3,5, and 6 weeks post-injury as well as the correlative histological changes were obtained. The RF-induced lesion was discrete with little spreading across the spinal cord. There was a good correlation between the histopathology findings and the MRI. We conclude that experimental RF lesioning of the spinal cord can produce a consistent lesion with predictable histopathological changes in experimental animals. A $65^{\circ} \mathrm{C}$ injury for $1 \mathrm{~min}$ induced a clinical picture of an incomplete SCI. The RF lesioning should be considered as a new model to study SCI, particularly those with a penetrating component.
\end{abstract}

Keywords: magnetic resonance imaging; spinal cord injury; radiofrequency

\section{Introduction}

Spinal cord injury (SCI) is an important and serious health problem in any country. Although relatively uncommon, ${ }^{1}$ personal, family, and social consequences can be deeply devasting. While motor vehicle accidents account for $40 \%$ of SCI, penetrating injuries, such as gunshot wounds, account for $13.6 \%{ }^{2}$

Many animal models have been utilized to study the pathophysiology of acute SCI. These models include cuff compression, ${ }^{3}$ screw compression, partial cord section, pharmacological blockade with ouabain, ${ }^{4}$ electromagnetic drivers, ${ }^{5}$ cryogenic injury, ${ }^{6}$ heat injury, ${ }^{7}$ and photochemically induced injury. ${ }^{8}$ Nevertheless, the most widely used methods are clip compression, ${ }^{9}$ and the weight-drop method. ${ }^{10}$ This latter technique is based on dropping a known weight from a known distance onto the exposed spinal cord. Nowadays, the weight-drop injury model has become sophisticated, incorporating sensors to assess the parameters of the impact and the biochemical responses of the tissue, ${ }^{11}$ or using a piston for a fine and consistent control of the impact force. ${ }^{12}$ However, the weight-drop model has shown a great variability in the degree of inflicted trauma related to the weight and the height of the dropped object and the weight of the impactor which rests against the spinal cord. In addition, the energy contained in the falling weight

Correspondence: Siavash S Haghighi, Room N 502, Division of Neurosurgery, One Hospital Drive, Columbia, Missouri 65212, USA at the time of the impact is not completely transformed into the traumatic energy transferred to the spinal cord. ${ }^{13}$

In this study we used radiofrequency $(\mathrm{RF})$ as a technique to induce injury in animals. The variables involved in this method, were time and intensity (voltage) which were fully controlled, hence creating a reproducible experimental SCI. The progression of the RF induced-injury was elevated on a weekly basis using the MRI ${ }^{14}$ which was correlated with the histological changes in each animal.

\section{Materials and methods}

Four female conditioned cats weighing $3.4 \mathrm{~kg}$ to $4.1 \mathrm{~kg}$ were anesthetized and ketamine (15 mg kg, IM) plus xylazine $(1.5 \mathrm{mg} \mathrm{kg})$, and atropine $(0.04 \mathrm{mg} / \mathrm{kg})$. To obtain a baseline MRI of the spinal cord, the cats were placed in a supine position on a wooden tray. To facilitate straight alignment of the spine, this tray was angulated at $45^{\circ}$ in the center. This procedure resulted in total immobilization of the body with minimal breathing motion, favoring a good magnetic signal acquisition. The images were taken by using a 1.5 tesla machine (Magnetom 42SP. 63SP, Siemens Medical Systems, Erlangen, West Germany). $\mathrm{T} 1 \quad(\mathrm{TR}=450$; $\mathrm{TE}=15)$, proton density $(\mathrm{TR}=2000 ; \mathrm{TE}=20)$, and $\mathrm{T} 2$ $(\mathrm{TR}=2000 ; \mathrm{TE}=90)$ images were obtained.

After the baseline MRI study, the cats were anesthetized using a halothane and oxygen mixture 
and were transferred to the operating table. They were placed in a prone position and their heads were fixed in a stereotactic frame (Model 1404, David Kopf Instruments, Tujunga, California). The body temperature of $36.5^{\circ} \pm 1 \mathrm{C}$ was maintained by placing the animals on a heating blanket (Aquamatic K-model and K-pad, Gorman Rupp Industries, Belteville, Ohio). The temperature was monitored by a rectal thermometer. The EKG was continously monitored using a standard physiograph. The right cephalic vein was cannulated for the administration of fluids.

Under a light halothane anesthetic, baseline cortical somatosensory evoked potentials (CSEPs) were recorded by placing the active needle electrode in the midline of the scalp. The reference needle electrode was placed in the midline nasal region. The analysis time was $50 \mathrm{msec}$. Low/high cutoff filters were set at 10 and $500 \mathrm{~Hz}$, respectively. The number of averages were 250 . The tibial nerve was stimulated by placing two needle electrodes transcutaneously in the plantar surface of the right hind paw adjacent to the nerve. An isolated stimulus $(0.2 \mathrm{msec}$ in duration at $2.81 \mathrm{~Hz})$ was given at twice motor threshold of 5 to 8 volts using an isolation unit. The CSEPs were recorded by a signal averager (Model 8400, Cadwell Laboratories Inc., Kennewick, Washington). At least two traces were obtained to ensure the reproducibility of cortical evoked responses. After the baseline CSEPs were recorded, and under sterile conditions, a T11-T12 laminectomy was performed under the microscope. The dura and the underlying dorsal columns at the T11-T12 segments were gently and vertically punctured using a 25-gauge needle. A $1 \mathrm{~mm}$ exposed tip RF electrode (Temperature monitoring electrode, Radionics, Burlington, Massachusetts) was vertically introduced at a low speed into the spinal cord using a mechanically driven micromanipulator to $1.5 \mathrm{~mm}$ depth. In one cat (animal \#3), the electrode was placed at $2.5 \mathrm{~mm}$ depth. A thermo-lesion at $65^{\circ} \mathrm{C}$ for 1 min was induced using an RF generator (Model RFG-3AV Lesion generator, Radionics, Burlington, MA). After the lesioning, the electrode was gently withdrawn and the epidural fat was placed on the punctured dura to prevent epidural adhesion, and the wound was closed in layers. Immediately after making the RF lesion, CSEPs were recorded. After the injury, animals recovered from the anesthesia. The urinary bladder was expressed in each animal four times a day until full recovery of spontaneous micturition occurred. The motor function of hind limbs were evaluated daily using the modified Tarlov scale, ${ }^{15}$ with the following grading parameters:

\section{No movement}

1 Barely perceptible movements in the hind limbs. No weight bearing

2 Frequent and vigorous movements in the hind limbs. No weight bearing
3 Can support weight with hind limbs. May take one or two steps

4 Walks with only mild deficit

5 Normal, but slow walking

6 Full and fast walking.

The MRI were repeated, at $2 \mathrm{nd}$, 3rd, 5th, and 6 th week post-injury (one cat at each week). Immediately after the second MRI, each animal was euthanized by transcardiac injection of sodium pentobarbital. Ten $\mathrm{mm}$ of spinal cord inclusive of the injured segment were immediately removed. The spinal cord segments were fixed in $10 \%$ buffered formalin. The spinal segments were transversely sectioned at 3-4 mm intervals and embedded in a single paraffin block. Serial sections were made at $8-10 \mu \mathrm{m}$ thickness utilizing a Reichert 2030 microtome. These sections were stained for Hematoxylin and Eosin. Tissue injury was assessed based on the presence of congestion, hemorrhage, edema, and tissue disruption. Neuronal injury was assessed by examination of the nuclear and cytoplasmic characteristics - cell shape, cytoplasmic density, eosinophilia, presence of Nissl substance and nuclear chromatin density.

\section{Results}

All of the cats became paraplegic, as was demonstrated by the loss of hindlimb function, and of bowel and bladder incontinence. Spontaneous micturition resumed in all of the animals after the 5th day postinjury. The CSEPs were abolished immediately after lesioning (Figure 1). By the 2nd week, gradual clinical recovery was observed, but no cat was able to walk. Only after the 5th and the 6th weeks, the animals could support their weight using their hind limbs and they started to take a few steps (Table 1).

The baseline (pre-injury) MRI of all animals showed good image quality, particularly in the

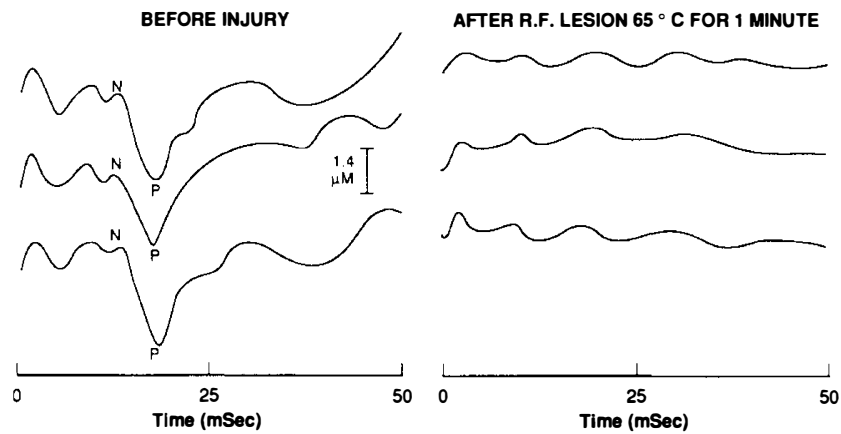

Figure 1 Cortical somatosensory evoked potentials (CSEPs) are recorded before (left column) and immediately after (right column) the radiofrequency lesioning at $65^{\circ} \mathrm{C}$ for $1 \mathrm{~min}$ in a cat. Complete loss of the evoked response is shown after this degree of injury 
Table 1 Motor function profiles of the spinal cord injured animals evaluated using the modified Tarlov scale*

\begin{tabular}{lccccccc}
\hline Animal\# & Baseline & Injury & 1st wk & 2nd wk & 3rd wk & 5th wk & 6th wk \\
\hline 1 & 6 & 0 & 1 & 2 & 2 & 3 & 3 \\
2 & 6 & 0 & 1 & 2 & 3 & 3 \\
3 & 6 & 0 & 1 & 2 & 2 & 3 \\
4 & 6 & 0 & 1 & 2 & & \\
\hline
\end{tabular}

*Modified Tarlov scale: 0, No movement; 1, Barley perceptible movements in the hind limbs. No weight bearing; 2 , Frequent and vigorous movements in the hind limbs. No weight bearing; 3, Can support weight with hind limbs. May take one or two steps; 4, Walks with only mild deficit; 5, Normal, but slow walking; 6, Full and fast walking. The MRI studies were taken at 2nd, 3rd, 5th, and 6th week post-injury (one cat at each week)

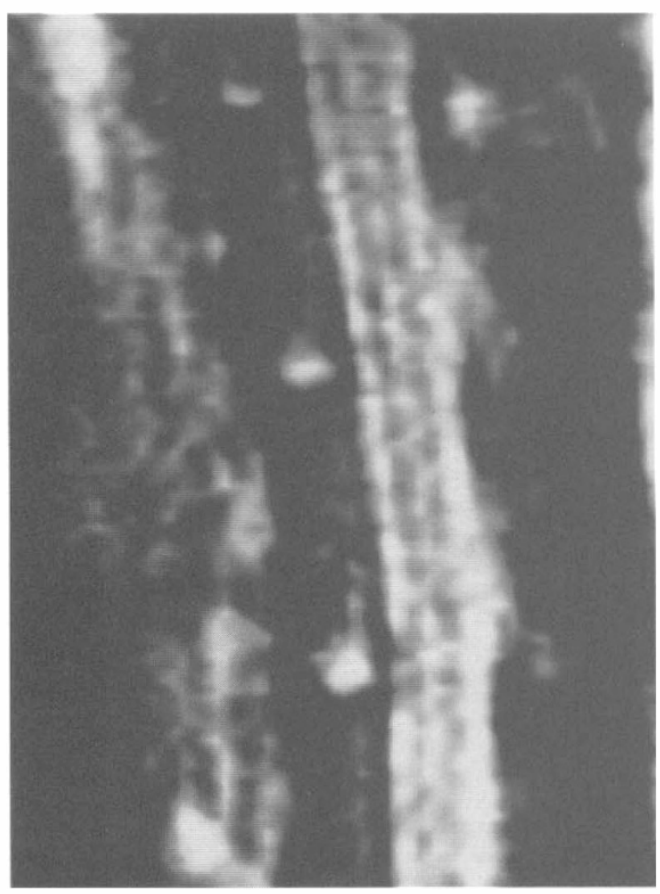

Figure 2 Sagittal T2 W. Midthoracic spine before the injury (baseline), showing good visualization of CSF around the cord as a homogeneous high signal. The spinal cord is well seen as a medium to high signal separated by a dark line from the CSF space

sagittal views using $\mathrm{T} 2 \mathrm{~W}$ with structural differentiation of the spinal cord, vertebral bodies, and epidural fat (Figure 2).

Two weeks post-injury, a penetrating transverse lesion was observed in a sagittal (T2 W) projections (Figure 3a) as a high signal focal area in the center of the spinal cord. Histologically, the electrode track was located at the injury site. The track did not reach the ependymal canal and the gray matter did not show any abnormality. However multiple small microcysts were seen in all of the white matter tissue (Figure $3 \mathrm{~b}$ ).

Three weeks post-injury, a T2 W sagittal projection depicted an irregular ovoid lesion as a high focal signal located in the dorsal and central region of the spinal cord (Figure 4a). Histologically, a deeper electrode track was observed corresponding to the

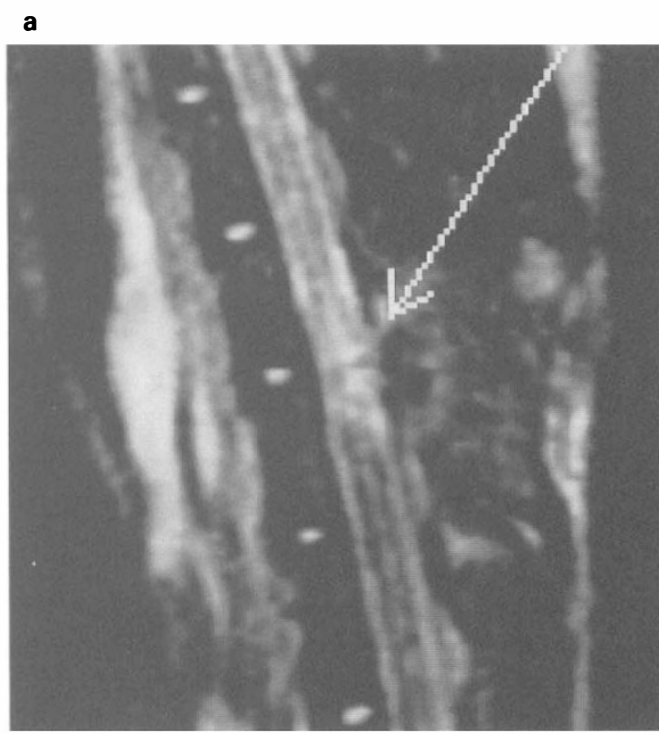

b

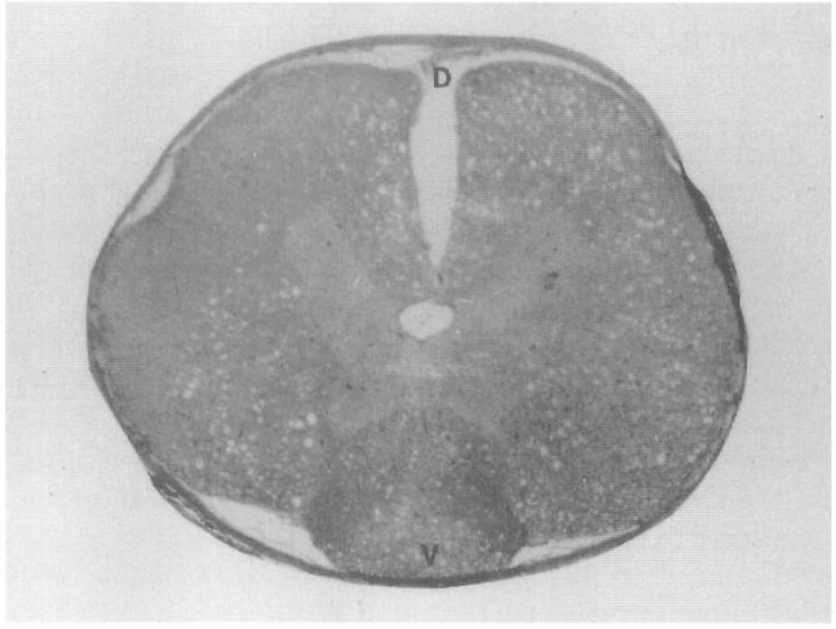

Figure 3 (a) Sagittal T2 W. Two weeks after the injury. The arrow points to the focal high signal lesion located at the site of injury. Margins are poorly marginated. (b) Two weeks after the injury. The histology of the spinal cord is shown with the radiofrequency electrode track at the site of the lesion. The track did not reach the ependymal canal and the gray matter did not show any abnormality. $\mathrm{D}=$ dorsal $\mathrm{V}=$ ventral 
deeper penetration of the electrode (animal \#3), which reached the ventral part of the spinal cord. Surrounding the track, necrotic changes affecting the central gray matter was notable. The remaining white matter showed demyelination and multiple small microcysts (Figure 4b).

Five weeks post-injury, a wide and more blunt cleft was observed in the sagittal (T2 W) projections with smaller intensity signal than the previous weeks (Figure 5a). The histopathological changes consisted of the necrotic areas in dorsal columns and central

a

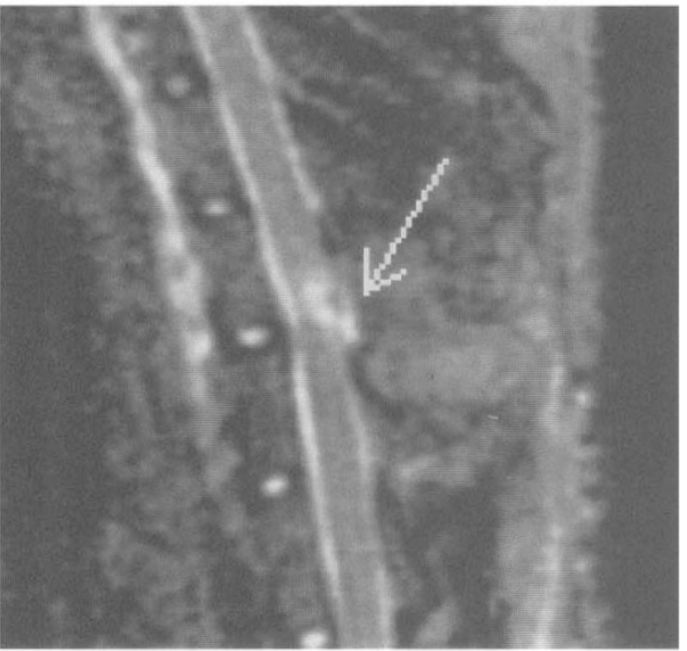

b

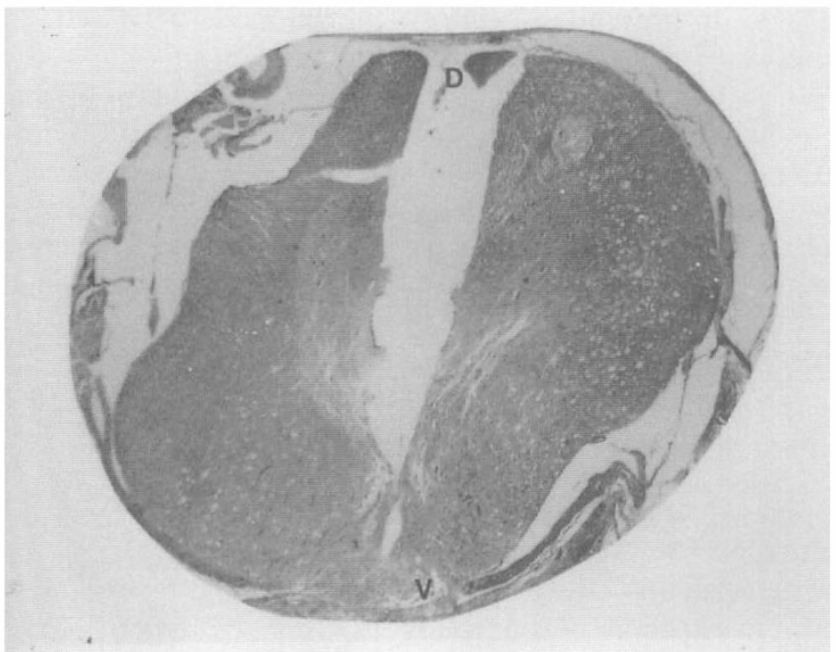

Figure 4 (a) Sagittal T2 W. Three weeks after the injury. The lesion, as a focal high signal area, is better delineated with more sharp margins. (b) Three weeks after the injury. Histology of the spinal cord at the site of the lesion showing a deeper electrode track corresponding to the deeper $(2.5 \mathrm{~mm}$ depth, animal \#3) penetration of the electrode in this animal. Surrounding the track, necrotic changes affecting the central matter is noticeable. The remaining white matter shows demyelination and multiple small microcysts. $\mathrm{D}=$ dorsal $\mathrm{V}=$ ventral gray matter with the existance of some irregular holes replacing the normal tissue. In the white matter many microcysts were found (Figure 5b).

Six weeks post-injury, the lesion was similar to the 5 th week lesion showing a wide and moderately high signal cleft in the sagittal views (Figure 6a). Histologically, a large hole was found in the center of the dorsal columns, surrounded by necrotic tissue spreading through the neighbouring gray matter. In the remaining white matter, microcysts and demyelination were more numerous and pronounced (Figure $6 \mathrm{~b}$ ).

a

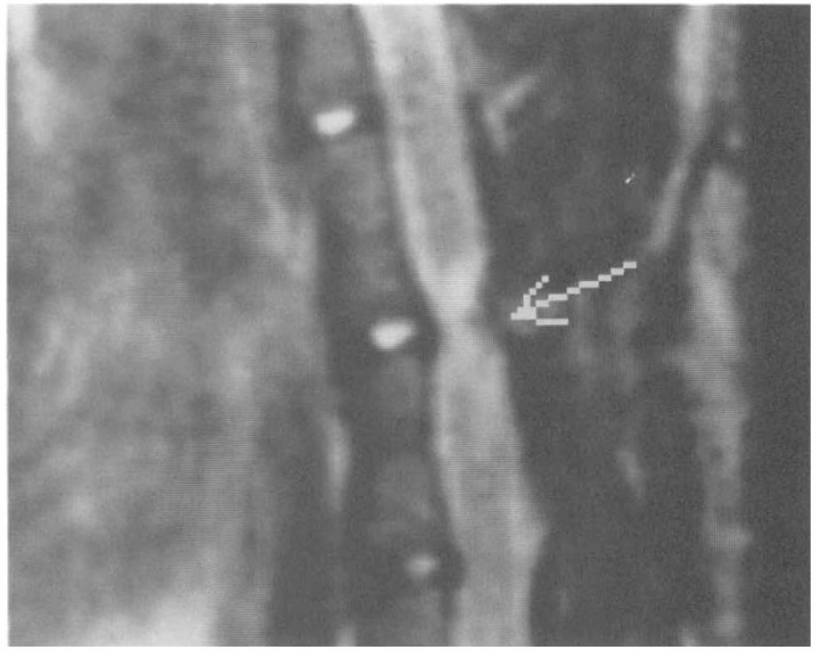

b

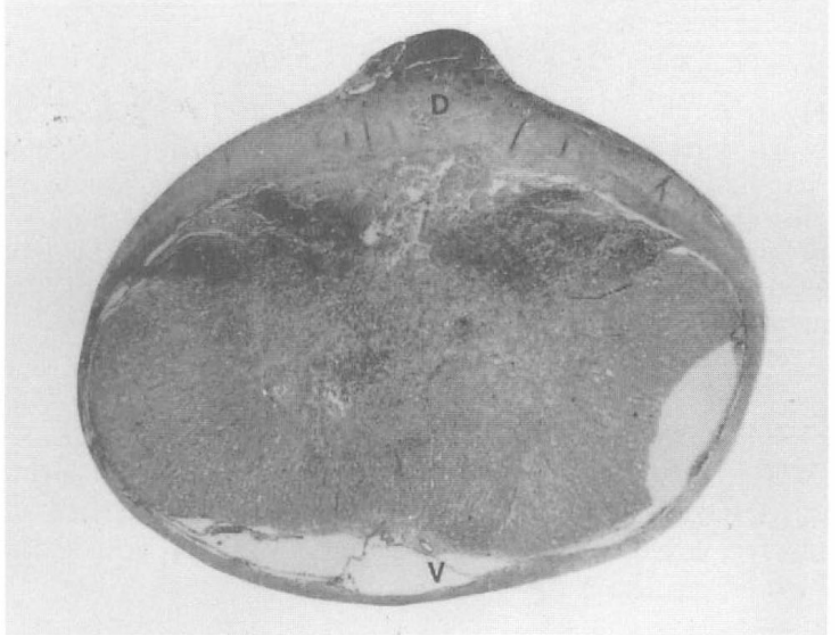

Figure 5 (a) Sagittal T2 W. Five weeks after the injury. Scar tissue is present with an apparent reduction in the sagittal diameter of the spinal canal possibly due to diminution of the CSF space. There is still a focal bright signal within the spinal cord at the site of injury. (b) Five weeks after the injury. Histology of the spinal cord at the site of injury showing necrotic areas in the dorsal columns and central gray matter with the existence of some irregular holes replacing the normal tissue. $\mathrm{D}=$ dorsal $\mathrm{V}=$ ventral 


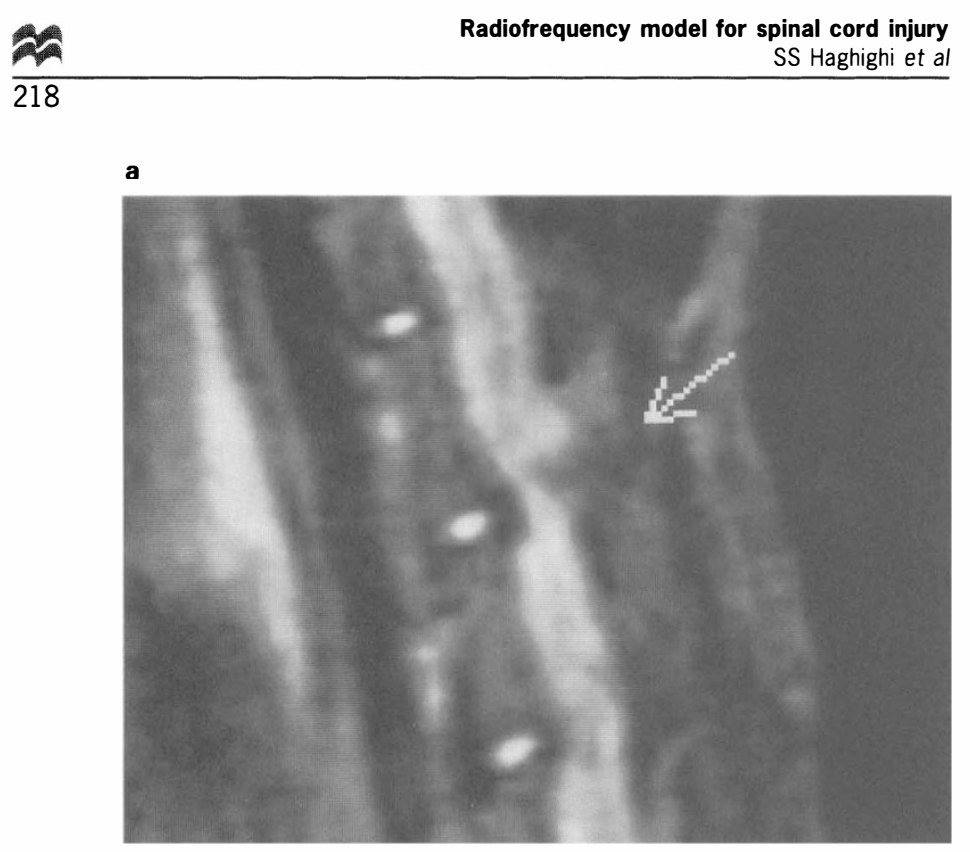

b

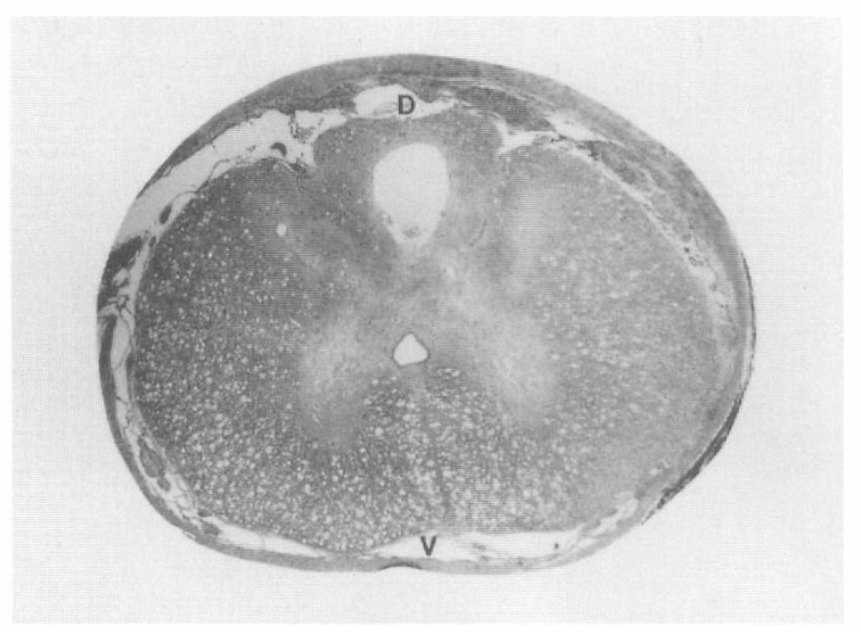

Figure 6 (a) Sagittal T2 W. Six weeks after the injury. The spinal cord is smaller in diameter and a focal area of high signal is difficult to visualize. (b) Six weeks after the injury. Histology of the spinal cord at the site of lesion showing the presence of a large hole in the center of the dorsal column, surrounded by necrotic tissue spreading through the neighbouring gray matter. $\mathrm{D}=$ dorsal $\mathrm{V}=$ ventral

No histological changes were found in the spinal cord tissue obtained one segment below the central injured zone, indicating the discretion of the lesion formed by the radiofrequency.

\section{Discussion}

After an acute SCI, two separate components of injury occur. These components, termed the primary ${ }^{16}$ and secondary ${ }^{17}$ injury contribute to the final neurological outcome.

Almost all models of experimental SCI develop an extended lesion which affects distant areas from the site of the initial injury. This is a major impediment towards experimental modelling for SCI. The problem of variability in the morphological changes occurring with these models limits the interpretation of changes associated with the injury. This problem is even more relevant in creating an injury model which applies to both blunt and penetrating injuries. For example, when the weight-drop technique was used to create a model for posttraumatic syrinx in four cats, only two animals developed central cavitation, while one animal showed only malacic changes without pathological evidence of cyst formation, and the fourth animal had a normal-appearing cord. ${ }^{18}$

MRI has proven its superior value in comparison with computed tomography or intraoperative sonography in diagnosing the intraparenchymal spinal cord lesion, with or without a 'radiological' abnormality. ${ }^{19-22}$ The changes affecting the spinal cord after a traumatic injury have been well studied using MRI. ${ }^{23-25}$ Weirich and co-workers have demonstrated good correlation between histological changes and the visual appearance of MR images in SCI. ${ }^{18}$

MRI has been used to study the pathological changes developing after a knife stab wound at the thoracic level. ${ }^{27} \mathrm{~T} 2$ images which were obtained 2 weeks after this injury demonstrated a bandlike lesion of increased signal. This lesion was representing an intraparenchymal spinal cord edema, and was associated with a good prognosis of neurological recovery. On the contrary, hypo-intense signal in $\mathrm{T} 2$ images have been correlated with oxidative changes of hemaglobin, which occurs 10 to 14 days after the injury. ${ }^{28}$ This usually specifies a severe hemorrhagic lesion with a poor neurological prognosis. ${ }^{24}$

As in previous studies, ${ }^{29,30}$ we have observed an increased signal in T2-weighted images in our study. We found that with the Tl-weighted method, the images appeared as an iso-intensity signal with a low diagnostic value not suitable in experimental SCI studies. In our model, which may resemble a penetrating injury, the RF-induced lesion was discrete with little spreading across other levels of the spinal cord. Meantime, there was a good correlation between the histopathological findings and the MRI.

Taking these into consideration, we believe that experimental RF lesioning of the spinal cord can produce a consistent lesion cross the lesion site which produce predictable histopathological changes. Furthermore, by changing the intensity (voltage) or time one can selectively induce incomplete or complete SCI in experimental animals. Further refinement of the technique by introducing sharper RF electrodes can minimize the problem of variability in experimental SCI.

\section{Acknowledgements}

It is a pleasure to thank Dr EH Adelstein, and Miss Barrett for excellent histological assistance. 


\section{References}

1 Tator CH, Duncan EG, Lapczak LI, Andrews DF. Changes in epidemiology of acute spinal cord injury from 1947 to 1981 . Surg Neurol 1993; 40: 207-215.

2 Burney RE, Maio RF, Maynard F, Karunas R. Incidence, characteristics and outcome of spinal cord injury at trauma centers in North America. Arch Surg 1993; 128: 596-599.

3 Martin D et al. Experimental acute traumatic injury of the adult rat spinal cord by a subdural inflatable balloon: methodology, behavioral analysis, and histopathology. J Neurosci Res 1992; 32: $539-550$

4 Schramm J, Krause R, Shigeno T, Brock M. Experimental investigation on the spinal cord evoked injury potential. $J$ Neurosurg 1983; 59: 485-492.

5 Stokes BT, Noyes DH, Behrmann DL. An electromechanical spinal cord injury technique with dynamic sensitivity. $J$ Neurotrauma 1992; 9: 187-195.

6 West NR, Collins GH. Cellular changes during repair of a cryogenic spinal cord injury in the rat: an electron microscopic study. J Neuropathol Exp Neurol 1989; 48: $94-108$.

7 Sasaki M, Ide C. Aberrant remyelination of axons after heat injury in the dorsal funiculus of rat spinal cord. Acta Neuropathol 1991; 81: $557-561$.

8 Watson BD et al. Photochemically induced spinal cord injury in the rat. Brain Res 1986; 367: 293-300.

9 Rivlin AS, Tator $\mathrm{CH}$. Effect of duration of spinal cord injury compression in a new acute cord injury model in the rat. Surg Neurol 1978; 10: $39-43$.

10 Allen AR. Surgery of experimental lesion of spinal cord equivalent to crush injury of fracture dislocation of spinal column. Preliminary report. JAMA 1911; 57: 878.

11 Gruner JA. A monitored contusion model of spinal cord injury in the rat. J Neurotrauma 1992; 9: $151-154$.

12 Blight A, Decrescito V. Morphometric analysis of experimental spinal cord injury in the cat: the relation of injury intensity to survival of myelinated axons. Neuroscience 1986; 19: $321-341$.

13 Panjabi MM. Experimental spinal cord trauma. A biomechanical viewpoint. Paraplegia 1987; 25: 217-220.

14 Hackney DB et al. Hemorrhage and edema in acute spinal cord compression: demonstration by MR imaging. Radiology 1986; 161: $387-390$.
15 Tarlov IM. Spinal cord compression studies III. Time limits for recovery after gradual compression in dogs. Arch Neurol Psychiat 1954; 71: $588-597$.

16 Hovda D, Becker D, Katayama Y. Secondary injury in acidosis. Neurotrauma 1992; 9(S1): S47-S60.

17 Tator $\mathrm{CH}$, Fehlings MG. Review of the secondary injury theory of acute spinal cord trauma: J Neurosurg 1991; 75: 15-26.

18 Cohen WA et al. Posttraumatic syrinx formation: experimental study. AJNR 1985; 6: $823-827$.

19 Goldberg AL et al. The impact of magnetic resonance on the diagnostic evaluation of acute cervicothoracic spinal trauma. Skelet Radiol 1988; 17: 89-95.

20 Matsumura A et al. Magnetic resonance imaging of spinal cord injury without radiologic abnormality. Surg Neurol 1990; 33: $281-283$.

21 Mirvis SE et al. Acute cervical spine trauma: evaluation with 1.5T MR imaging. Radiology 1988; 166: 807-816.

22 Quencer RM et al. Magnetic resonance imaging of the chronically injured cervical spinal cord: $A J N R$ 1986; 7: 457-464.

23 Bondurant FJ et al. Acute spinal cord injury. A study using physical examination and magnetic resonance imaging. Spine 1990; 15: $161-168$

24 Kulkarni MV, Bondurant FJ, Rose SL, Narayana PA. 1.5 tesla magnetic resonance imaging of acute spinal trauma. RadioGraphics 1988; 8: 1059 - 1082.

$25 \mathrm{Kulkarni}$ MV et al. Acute spinal cord injury: MR imaging at 1.5T. Radiology 1987; 4: 837-843.

26 Weirich SD et al. Histopathological correlation of magnetic resonance imaging signal patterns in a spinal cord injury model. Spine 1990; 15: $630-638$

27 Mayer SJ, Kulkarni MV. MR Imaging of incisional spinal cord injury. AJNR 1987; 8: $925-927$.

28 Edelman RR, Warach S. Magnetic resonance imaging (Part I). $N$ Engl J Med 1993; 328: 708 - 716.

29 Kadoya S, Nakamura T, Kobayashi S, Yamamoto I. Magnetic resonance imaging of acute spinal cord injury. Report of three cases. Neuroradiology 1987; 29: $252-255$.

30 Schouman-Claeys $\mathrm{E}$ et al. MR imaging of acute spinal cord injury: results of an experimental study in dogs. $A J N R$ 1990; 11: $959-965$. 